\title{
A Modified Design of Class-E Power Amplifier with Balanced FETs and High Output Power for RFID Applications
}

\author{
Muhammad Noaman Zahid, Jianliang Jiang ${ }^{*}$, Heng Lu, Hengli Zhang \\ School of Optics and Photonics, Beijing Institute of Technology, Beijing, China \\ Received 06 April 2021; received in revised form 12 May 2021; accepted 13 May 2021 \\ DOI: https://doi.org/10.46604/peti.2021.7442
}

\begin{abstract}
In Radio Frequency (RF) communication, a Power Amplifier (PA) is used to amplify the signal at the required power level with less utilization of Direct Current (DC) power. The main characteristic of class-E PA is sturdy nonlinearity due to the switching mode action. In this study, a modified design of class-E PA with balanced Metal Oxide Semiconductor Field Effect Transistors (MOSFETs) and high output power for Electronic Article Surveillance (EAS) Radio Frequency Identification (RFID) application is presented. MOSFETs are adjusted to have high output performance of about $80 \%$ for RFID-based EAS system. A matching network is also proposed for accurate matching because there are differences in the behavior between RF waves and low frequency waves. The design of a matching network is a tradeoff among the complexity, adjustability, implementation, and bandwidth for the required output power and frequency. The implemented PA is capable of providing $44.8 \mathrm{dBm}$ output power with Power-Added Efficiency (PAE) of $78.5 \%$ at $7.7 \mathrm{MHz}$ to $8.7 \mathrm{MHz}$.
\end{abstract}

Keywords: class-E power amplifier, RFID applications, high output power, matching network, balanced FET

\section{Introduction}

In communication systems, Radio Frequency (RF) Power Amplifier (PA) is a key module of a transmitter. Therefore, it has been extensively studied for several eras [1-3]. The RF signal is fed to PA through an RF mixer for amplification, and then PA sends the amplified signal to the antenna through a band pass filter [4-7]. In comparison with the linear PA, switching PA has quite higher efficiency which theoretically achieves almost $100 \%$ and is most preferable for portable wireless communication systems. In the class-E function, the transistor behaves like a switch, thus the individual class-E PA is not proper for providing gain to Amplitude Modulated (AM) signal. However, for a fixed cover signal with simply phase information, class-E PA can be practical with academic drain efficiency as high as $100 \%$. Moreover, class-E PA is usually occupied in Envelope Elimination and Restoration (EER) technique [8-10]. In class-E PA, no energy is wasted as heat in the transistors because there is no overlapping in drain voltage and drain current. The main characteristic of class-E PA is the sturdy nonlinearity due to the switching mode action.

Although class-A, B, and C do not utilize rigid linear gain performance, the magnitude of input and output signals are relatable. Push-pull class-D voltage switching and current switching stages use series tuned resonant circuits or parallel tuned resonant circuits respectively, driven by two on-off switches [11-12]. In switching operation, when one transistor is ON and the other transistor is OFF at the same time, each transistor (switch) is in ON-state for half of the input signal. Therefore, push-pull amplifiers are efficient because of the switching transition, and there is a loss of efficiency at high frequencies [12]. It has been

* Corresponding author. E-mail address: jiangjianliang@ bit.edu.cn Tel.: +861-3-521656801; Fax: +86-1068914846 
observed that class-C PA works for less than a half cycle, and has higher noise effects and distortion [13]. Although such type of amplifiers has good efficiency, it suffers from poor dynamic range. In the case of a class-D PA, which is also recognized as a switching amplifier, it has less power loss because the active devices are kept fully off or fully on [14]. The class-E PA is more considerable for RF PAs designing because these amplifiers have higher efficiency than class-D and class-F [15]. In the case of class-F amplifier, drain current and drain voltage never overlap with each other [16].

In this study, a theoretical analysis and complete design of class-E PA with balanced Filed Effect Transistors (FETs) for high output power and high efficiency of Radio Frequency Identification (RFID) application is presented. The proposed PA is designed for 7.7 MHz to 8.7 MHz. The main purpose of the presented PA is to maximize Power-Added Efficiency (PAE) and enhance the output power at desirable levels. The remaining composition of the study is as follows. Section 2 presents a brief summary of the performance estimation factors of class-E PA. Section 3 deals with a brief discussion on the proposed circuit operation of the PA and matching network. Section 4 presents the results and analysis of the proposed PA. Finally, in section 5, the conclusion drawn from this research is summarized.

\section{Performance Estimation Factors of Class-E PA}

For microwave engineers, it remains challenging to design RF amplifiers with high efficiency and high power. In this section, the performance estimation factors are summarized. Some important factors are considered for designing class-E PA, which includes linearity, output power, power gain, PAE, and Power Utilization Factor (PUF).

\subsection{Linearity}

When a transistor is used for amplification purposes in PA because of its nonlinear behavior, there will be a distortion component, namely Inter Modulation Distortion (IMD), at the output of PA. For characterization of PA's linearity, 3rd order IMD is mostly used and expressed as:

$$
\begin{aligned}
& I M D 3_{L}=P_{o}\left(f_{1}\right)-P_{o}\left(2 f_{1}-f_{2}\right) \\
& I M D 3_{H}=P_{o}\left(f_{2}\right)-P_{o}\left(2 f_{2}-f_{1}\right)
\end{aligned}
$$

\subsection{Output power}

The output power can be explained as the power which is transmitted to the antenna or towards the load of the PA at the tuned or selected frequencies. If the load at given frequencies is purely resistive, a generic resistor $R_{L}$ can be used to describe the load. Using a matching network after the amplifier stage, the load impedance can be converted into higher to lower and lower to higher configurations.

Based on the above considerations, it can carry out power output basic calculations as:

$$
\begin{aligned}
& P_{T, \text { inst }}=V_{\text {out }}(t) I_{\text {out }}(t) \\
& P_{T, \text { avg }}=\lim _{t \rightarrow \infty} \frac{1}{t} \int_{-t / 2}^{t / 2} P_{T, \text { inst }}(t) d t \\
& P_{T}(A)=\frac{A^{2}}{2 R_{L}}=\frac{V_{T, \max }^{2}}{2 R_{L}}=\frac{V_{T, r m s}^{2}}{2 R_{L}}
\end{aligned}
$$


where $P_{T, \text { inst }}$ is the instantaneous power at any time $(t)$ with the average output power $P_{T, \text { avg }}$ as shown in Eqs. (3) and (4) respectively. If $V_{T, \max }$ defined in Eq. (5) is the sinusoidal wave having amplitude $(A)$ at the fundamental or tuned frequency and $V_{T, r m s}$ is the RMS voltage value corresponding to that frequency, then $P_{T}$ defined in Eq. (5) is the power amplified and generated by the PA at the fundamental frequency.

\subsection{Power Gain}

Let us consider that $P_{I N}$ is the RF power applied to the input of the circuit and $P_{T}$ is the power at output, then $P_{I N}$ and $P_{T}$ can be related to define the power gain $(G)$ of the PA as the ratio of $P_{I N}$ and $P_{T}$. The gain of the PA is usually expressed in dB as depicted in Eq. (6).

$$
G_{d B}=10 \log _{10}\left(\frac{P_{T}}{P_{I N}}\right)
$$

\subsection{Power-Added Efficiency (PAE)}

Efficiency is one of the most considerable and fundamental characteristics of RF PA. An efficient PA not only lowers down the heat dissipation but also reduces power consumption. The power conversion from Direct Current (DC) to the RF power is characterized by amplifier Drainage Efficiency (DE), which is defined as the ratio between amplified power at the output of PA $\left(P_{T}\right)$ and the total DC power consumed by the last of the total stages of PA in a circuit $\left(P_{d c, \text { drain }}\right)$ and is expressed in Eq. (7). It is noted that the drain output for bipolar devices is referred to as the collector efficiency. If $P_{I N}$ is considered the input power driving each stage of PA and $P_{d c, \text { drain }}$ consumed DC power, then another parameter related to efficiency which is called PAE must be introduced, and it can be expressed by the parameters $P_{T}$ and $P_{I N}$ as their difference in ratio with total power (DC) $P_{d c}$ consumed by PA. The total DC power $P_{d c}$ consumed is then defined as the sum of the total consumed DC power at the drain and the consumed DC power at a different section or stages ( $A k$ ) of the PA and is expressed in Eq. (8).

$$
\begin{aligned}
& D E=\frac{P_{T}}{P_{d c, \text { drain }}} \\
& P A E=\frac{P_{T}-P_{I N}}{P_{d c}}=\frac{P_{T}-P_{I N}}{P_{d c}+\sum_{k=1}^{n} P_{d c, A k}}
\end{aligned}
$$

\subsection{Power Utilization Factor (PUF)}

In PAs, the high frequency transistors are the most expensive components, and the maximum utilization of device capability is accordingly desirable. PUF refers to a measure of effective use of the device capability (reference is made to the operation of the same device in class mode) and is expressed in Eq. (9):

$$
P U F=\frac{P_{o(\text { class under study })}}{P_{o(\text { class } A)}}
$$

\section{Design of Class-E PA}

In microwave communication, a balanced amplifier is extensively used because of its improved stability as compared with a single ended amplifier. A balanced amplifier has lower noise and better input and output return losses than a single ended amplifier. In the case of one branch failure, it reduces the performance operation of PA. Noise figure and gain will 
degrade about $3 \mathrm{~dB}$ only instead of completely out of service. The proposed structure of class-E PA with balanced FETs is shown in Fig. 1. This work proposes a compromised topology that achieves high output power and high efficiency. Fig. 1 shows a structure of balanced amplifier where two similar single ended amplifiers are connected in parallel in each branch. Transistors $\mathrm{M}_{1}$ and $\mathrm{M}_{2}$ are used for RF signal amplification. $\mathrm{L}_{1}$ and $\mathrm{L}_{2}$ are RF chokes which are used for isolation of RF signal and VDD and allow only DC signal. The amplified signal of each single ended amplifier is then combined by a power combiner network. To achieve higher output power from a PA, several active devices are used in parallel or push-pull configuration. The transistors $\mathrm{Q}_{1}$ and $\mathrm{Q}_{2}$ and the transistors $\mathrm{Q}_{3}$ and $\mathrm{Q}_{4}$ serve as two separate gate driving circuits for $\mathrm{M}_{2}$ and $\mathrm{M}_{1}$ respectively. At higher frequencies, gate driver circuits are used for proper switching at Metal Oxide Semiconductor Field Effect Transistors (MOSFET). In this work, a square wave is used as an input signal to drive PA. For designing the gate driving circuits, the frequency of the driving signal is the most considerable factor. For this case, it needs to get the switching speed which is about $100 \mathrm{~ns}$.

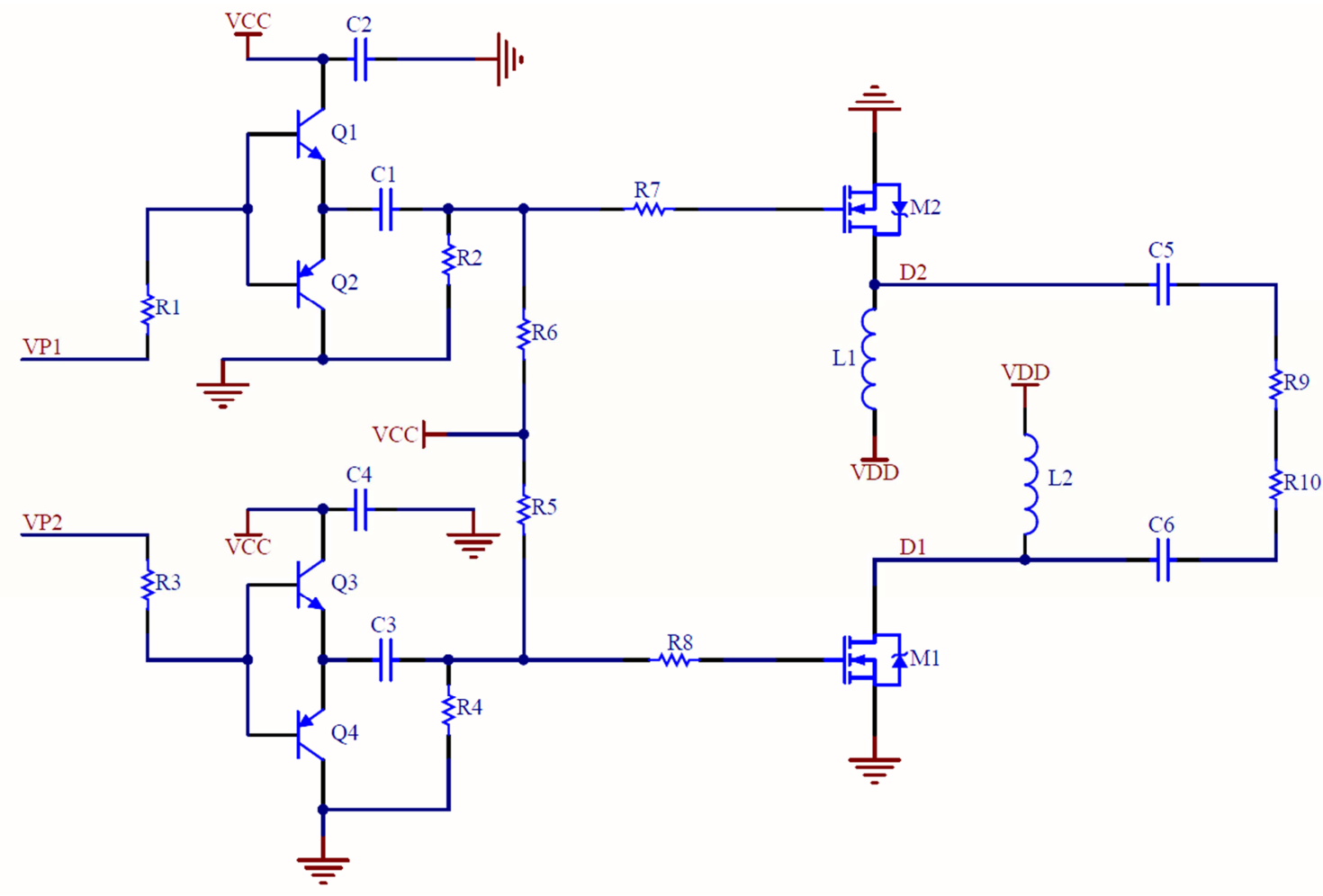

Fig. 1 The proposed class-E PA

Most PAs in RF ranges are specified to the transformation of power into a load containing an input impedance of $50 \Omega$. It can be designed easily a perfect matching network at precisely one frequency. However, since transmission lines and lossless matching networks are designed with the help of reactive elements, it is observed that the change of frequency is the cause of mismatch of the circuit. There are two kinds of effects of an inductor's parasitic resistance that can affect the ideal power transformation, i.e., the mismatches of the impedance and direct power loss of the network. Therefore, some power of the RF signal is reflected and cannot reach the output. Here network reflected power and power loss can be denoted as $P_{\text {ref }}$ and $P_{\text {loss }}$ respectively. Also, the power available from the source (VS) can be written as $P_{A V}$, the input power at the matching network can be written as $P_{i n}$, and the output power loss at $R_{L}$ is denoted as $P_{\text {out }}$. Thus, because of energy conservation, the expression is as given in Eqs. (10) and (11).

$$
\begin{aligned}
& P_{A V}=P_{i n}+P_{r e f} \\
& P_{i n}=P_{\text {out }}+P_{\text {loss }}
\end{aligned}
$$


The proposed matching network is depicted in Fig. 2(a). To obtain the frequency response as shown in Fig. 2(b), linear networks are used which are also known as band-pass or band-stop filters. In this case, by transforming the poles and zeros of the network, the filter is designed. First, the normalized approximated values are selected for inductors and capacitors at the required $\mathrm{N}$ ( $\mathrm{N}$ is the order of the filter). Then, the normalized band-pass frequency variable $\left(\Omega_{b p}\right)$ from the given Eq. (12) is evaluated.

$$
\Omega_{b p}=\frac{B W}{f_{r}}
$$

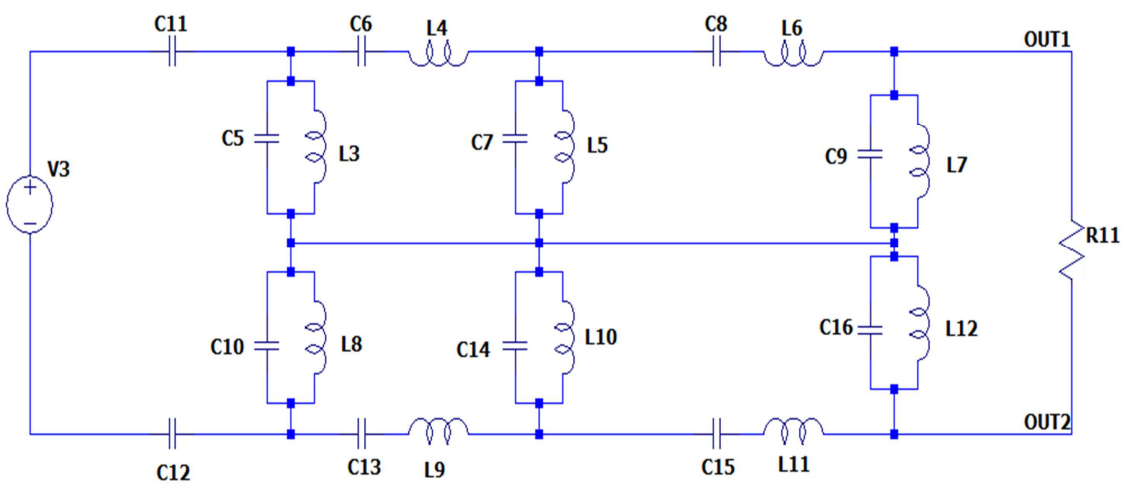

(a) Impedance transforming band-pass filter

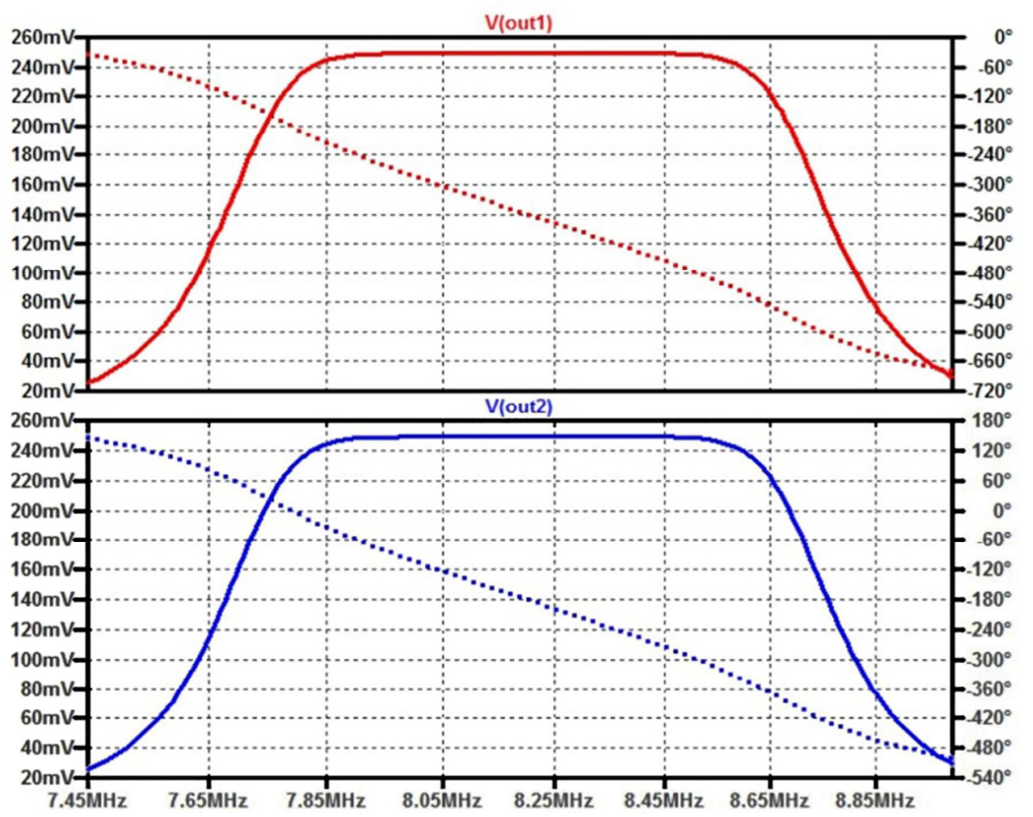

(b) Frequency response of matching network

Fig. 2 The proposed matching network and it's response

By applying the transformation of the approximated low pass values $\left(L_{n} C_{n}\right)$ to the band-pass LC section, the prepared series and shunt pairs $\left(L_{b} C_{b}\right)$ are expressed as Eqs. (13) and (14).

$$
\begin{gathered}
L_{b}=\frac{L_{n}}{\Omega_{b p}} \\
C_{b}=\frac{\Omega_{b p}}{L_{n}}
\end{gathered}
$$




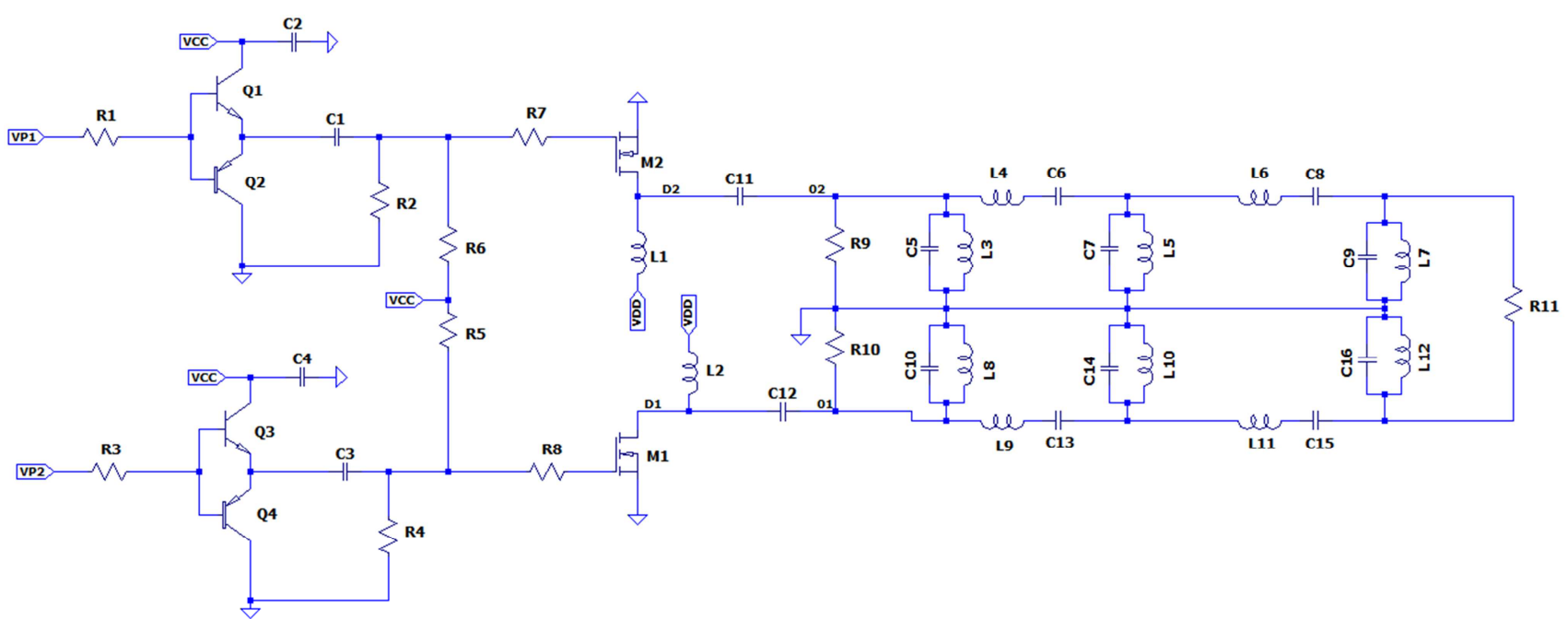

Fig. 3 The proposed class-E PA module with matching network

The balanced amplifiers are more stable as compared to single ended PAs. The complete schematic of the proposed class-E PA module with matching network is depicted in Fig. 3. The actual load at both ends (input and output) of PA will not affect the load to the branch amplifiers too much. While the single ended amplifier is loaded with actual load and the amplifier may be unstable for some frequencies ranges. In addition, the balanced amplifiers have more redundancy functions and high reliability. The balanced amplifiers have some disadvantages which are:

(1) Difficult integration because the component counts, power divider, and power combiners are doubled.

(2) Balanced amplifiers are expensive because more components are used.

However, due to the higher stability and high performance, balanced amplifier is the best choice for RF and microwave engineers for high end amplification in RF/microwave infrastructure applications. Advantages and disadvantages of a balanced amplifier in comparison with single ended amplifier are summarized in Table 1:

Table 1 Summary of advantages and disadvantages of the balanced amplifier

\begin{tabular}{|c|c|c|}
\hline Parameter & Balanced amplifier & Single ended amplifier \\
\hline Input/output return loss & Excellent & Fair \\
\hline Size & Large & Small \\
\hline Reliability & 2 time higher & - \\
\hline Performance stability in temperature & Excellent & Poor \\
\hline Performance stability with component & Excellent & Poor \\
\hline Unconditional stable & Easy & Difficult \\
\hline Cost & Expensive & Cheaper \\
\hline Integration & Difficult & Excellent \\
\hline
\end{tabular}

\section{Results and Discussion}

The proposed PA is designed by using Altium designer and LTspice circuit simulator. The performance of PA can be affected by the parasitic components. In the proposed design, no energy is wasted as heat in the transistors because there is no overlapping in drain voltage and drain current. The voltage and current waveforms (Figs. 4 (a) and (b)) assure the high efficiency of the power module.

Fig. 5(a) depicts the simulated results of Signal Integrity (SI) analysis which is performed by using Altium designer. The PCB layout of the proposed power module is given in Fig. 5(b). SI refers to the quality of signal on the track, and is able to respond with the accurate timing and voltage in the circuit. In the circuit design, the normal operation of any device can be affected by SI issues. These issues contain overshoot, reflection, cross talk, delay and timing errors, EM radiation, and some other factors. 


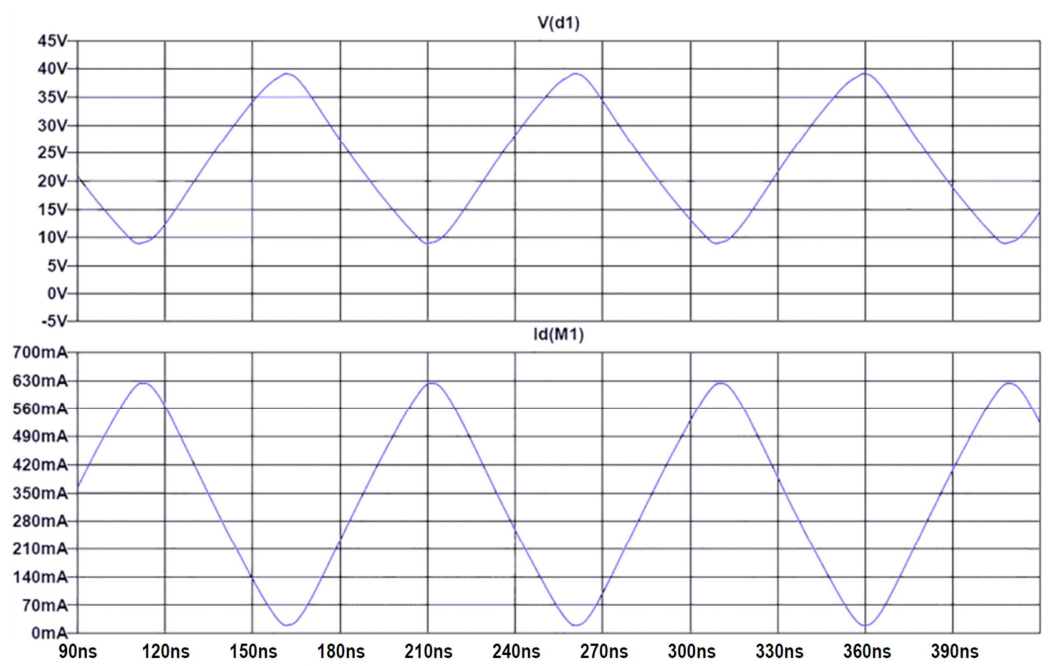

(a) MOSFET M1

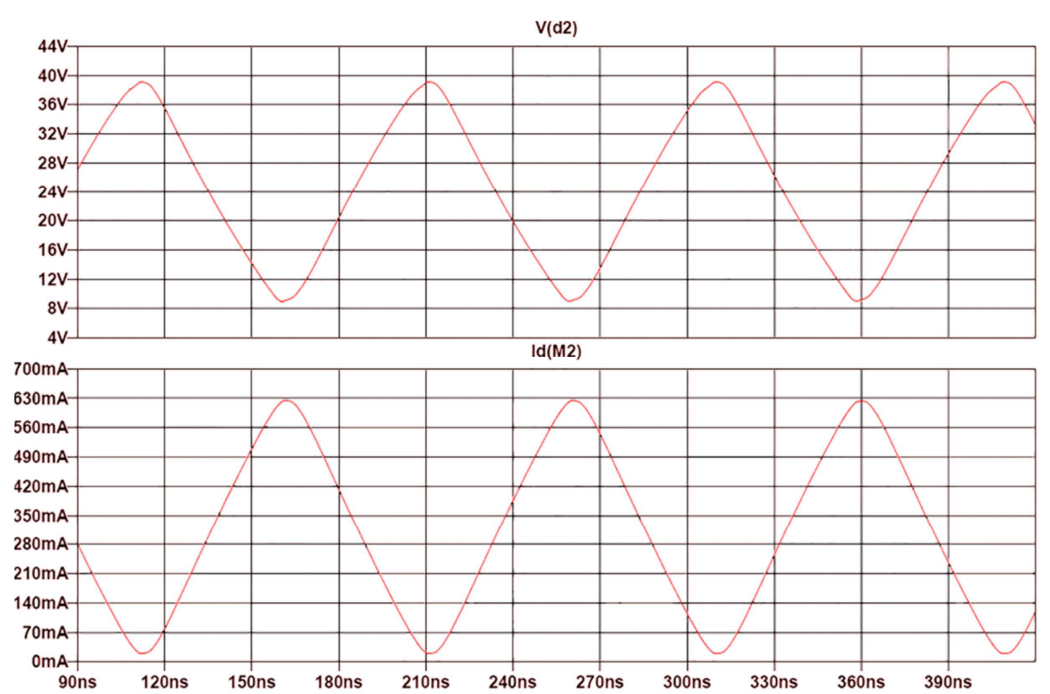

(b) MOSFET M2

Fig. 4 Simulated waveforms of drain voltages and drain currents

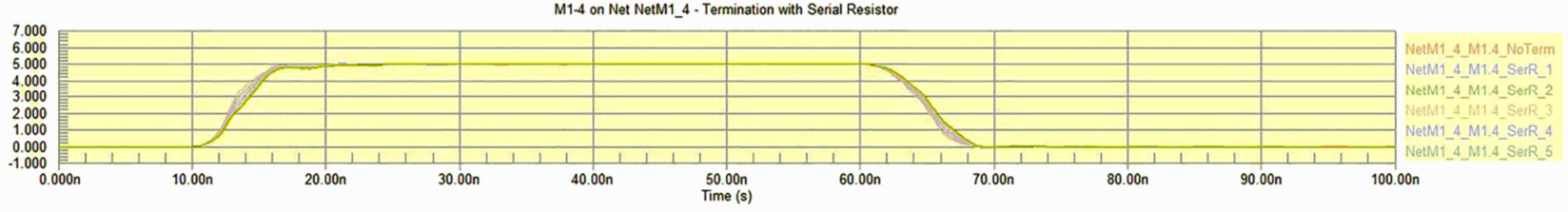

M2-1 on Net NetM1_4 - Termination with Serial Resistor
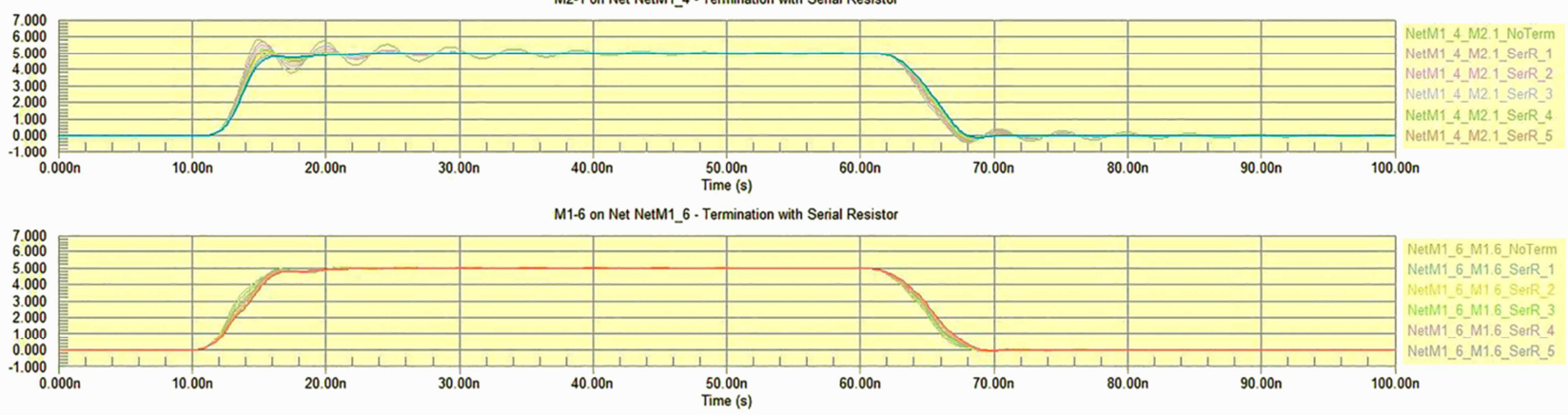

M2-2 on Net NetM1_6 - Termination with Serial Resistor

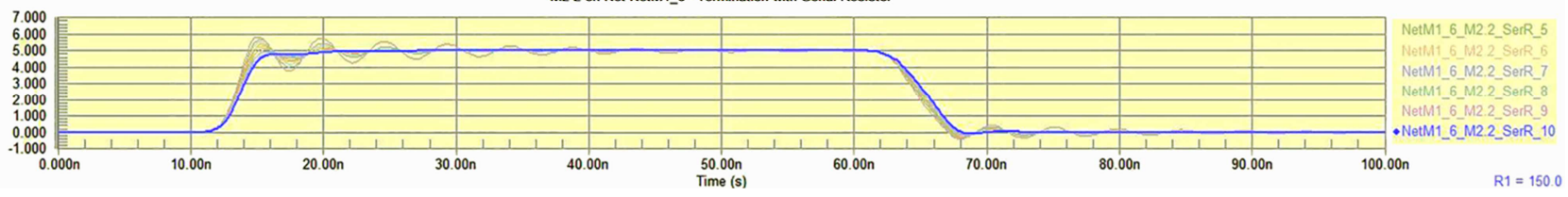

(a) Signal integrity analysis

Fig. 5 Signal integrity analysis of the proposed PA 


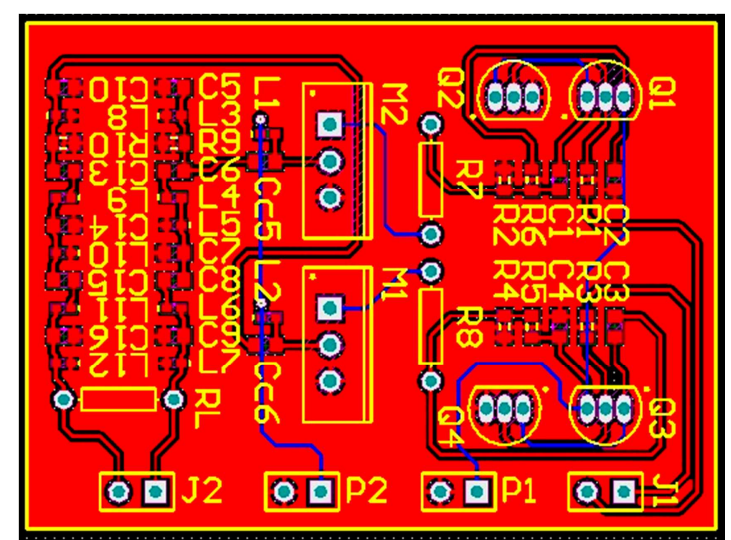

(b) PCB layout of PA module

Fig. 5 Signal integrity analysis of the proposed PA (continued)

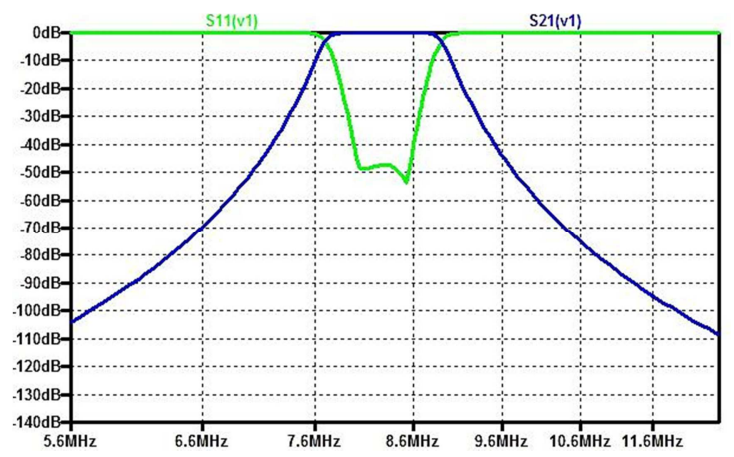

Fig. 6 Insertion and return loss characteristics

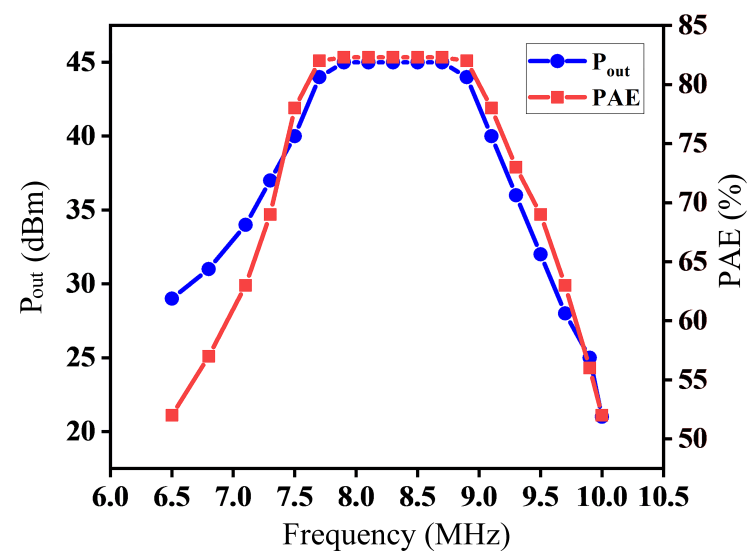

Fig. $7 \mathrm{P}_{\text {out }}$ and PAE versus frequency

It can be seen from Fig. 6, the return and insertion loss shows the effective performance of the band-pass matching network. Fig. 7 illustrates the output power and PAE performance versus frequency. It can be seen that the output power is stable during the desired frequency band.

Finally, we compare the performance of the proposed amplifier with previously reported work, as shown in Table 2 . The topology of the previous work is similar but used for different applications.

Table 2 Performance analysis with previous work

\begin{tabular}{|c|c|c|c|c|}
\hline Ref. & Frequency & $\mathrm{P}_{\text {out }}(\mathrm{dBm})$ & PAE $(\%)$ & Application \\
\hline$[17]$ & $2.14 \mathrm{GHz}$ & 46.02 & 67.4 & WCDMA base station \\
\hline$[18]$ & $2.5 \mathrm{GHz}$ & 21.46 & 64 & Low power applications \\
\hline$[19]$ & $1.5-2 \mathrm{GHz}$ & 30.7 & 45.6 & - \\
\hline$[20]$ & $1850-1910 \mathrm{MHz}$ & 28 & 41 & 3G handset application \\
\hline This work & $7.7-8.7 \mathrm{MHz}$ & 44.8 & 78.5 & EAS RFID application \\
\hline
\end{tabular}




\section{Conclusions}

In this study, a modified balanced MOSFET class-E PA with high output power and high efficiency for RFID-based EAS system is analyzed and implemented. The proposed power module is designed and simulated by using MOSFETs from Vishy siliconix for the frequency band of $7.7 \mathrm{MHz}$ to $8.7 \mathrm{MHz}$. The results demonstrate a saturated output power of $44.8 \mathrm{dBm}$ and a PAE of $78.5 \%$ at desired frequency operation. Additionally, the proposed matching network transforms the maximum power towards the load. From the results, it is verified that the proposed power module can deliver higher output power and higher efficiency performance than the amplifier using single ended topology for RFID applications.

\section{Conflicts of Interest}

The authors declare no conflict of interest.

\section{References}

[1] S. Cripps, RF Power Amplifiers for Wireless Communications, 2nd ed. Norwood: Artech House, 2006.

[2] J. C. Du, Z. G. Wang, J. Xu, X. Chen, and T. Z. Qin, “A Differential Class-E Power Amplifier with Dynamic Body Bias Technique," Microwave and Optical Technology Letters, vol. 62, no. 1, pp. 130-136, September 2019.

[3] P. Colantonio, F. Giannini, and E. Limiti, High Efficiency RF and Microwave Solid State Power Amplifiers, United States: John Wiley \& Sons, 2009.

[4] J. Pang, Y. Li, Y. Zhang, X. Y. Zhou, Z. Dai, and A. Zhu, "Analysis and Design of Highly Efficient Wideband RF-Input Sequential Load Modulated Balanced Power Amplifier,” IEEE Transactions on Microwave Theory and Techniques, vol. 68, no. 5, pp. 1741-1753, May 2020.

[5] I. Casallas, C. Páez, A. Fajardo, and G. Perilla, "Design Methodology of Class-E Power Amplifier Using Feed Inductance Tuning for High-Efficiency Operation in Wireless Power Transfer Applications,” IEEE ANDESCON, October 2020, pp. $1-6$.

[6] M. N. Zahid, J. Jiang, U. Rafique, and D. Eric, "Modified Planar Square-Loop Antenna for Electronic Article Surveillance Radio Frequency Identification Applications,” Journal of Communication Technology and Electronics, vol. 65, no. 10, pp. 1161-1166, October 2020.

[7] P. Afanasyev, A. Grebennikov, R. Farrell, and J. Dooley, "Broadband Operation of Class-E Power Amplifier with Shunt Filter," 18th IEEE International New Circuits and Systems Conference, June 2020, pp. 54-57.

[8] F. Moloudi and H. Jahanirad, "Broadband Class-E Power Amplifier Design Using Tunable Output Matching Network," AEU-International Journal of Electronics and Communications, vol. 118, 153142, 2020.

[9] M. E. Nouri, S. Roshani, M. H. Mozaffari, and A. Nosratpour, "Design of High-Efficiency Compact Doherty Power Amplifier with Harmonics Suppression and Wide Operation Frequency Band," AEU-International Journal of Electronics and Communications, vol. 118, 153168, May 2020.

[10] M. N. Zahid, J. Jiang, H. Lu, S. Khan, and H. Zhang, “An Optimal Design of High Output Power CMOS Class E Power Amplifier with Broadband Matching for RFID Applications,” Journal of Physics: Conference Series, vol. 1746, no. 1, 012089, January 2021.

[11] H. Lyu and K. Chen, "Balanced-to-Doherty Mode-Reconfigurable Power Amplifier with High Efficiency and Linearity Against Load Mismatch,” IEEE Transactions on Microwave Theory and Techniques, vol. 68, no. 5, pp. 1717-1728, May 2020.

[12] O. V. Varlamov and V. N. Gromorushkin, "Class D Switching Power Amplifier with a Filter under Load Mismatch Conditions," Wave Electronics and Its Application in Information and Telecommunication Systems, June 2020, pp. 1-6.

[13] H. T. Nguyen and H. Wang, "A Coupler-Based Differential mm-Wave Doherty Power Amplifier with Impedance Inverting and Scaling Baluns," IEEE Journal of Solid-State Circuits, vol. 55, no. 5, pp. 1212-1223, May 2020.

[14] W. Cai, L. Huang, and S. Wang, “Class D Power Amplifier for Medical Application,” Informatics Engineering, an International Journal, vol. 4, no. 2, pp. 9-15, June 2016.

[15] Z. Zhang, Z. Cheng, H. Ke, G. Liu, and S. Li, "Design of a Broadband High-Efficiency Hybrid Class-EFJ Power Amplifier," IEEE Microwave and Wireless Components Letters, vol. 30, no. 4, pp. 407-409, April 2020.

[16] C. Pakasiri, P. Manasummakij, and S. Wang, "Modified Class-F power Amplifier Design with Fundamental Frequency Output Impedance Load,” AEU-International Journal of Electronics and Communications,vol. 132, 153637, April 2021. 
[17] Y. S. Lee, M. W. Lee, and Y. H. Jeong, “A 40W Balanced GaN HEMT Class-E Power Amplifier with 71\% Efficiency for WCDMA Base Station,” Microwave and Optical Technology Letters, vol. 51, no. 3, pp. 842-845, March 2009.

[18] F. Tamjid, Y. Alekajbaf, J. Y. Hasani, and A. Rahmati, “Analysis and Design a 2.5 GHz Class-E Power Amplifier in Two Configurations," 5th Iranian Conference on Electrical and Electronics Engineering, August 2013, pp. 187-192.

[19] O. Lee, J. Han, K. H. An, D. H. Lee, K. S. Lee, S. Hong, et al., "A Charging Acceleration Technique for Highly Efficient Cascode Class-E CMOS Power Amplifiers,” IEEE Journal of Solid-State Circuits, vol. 45, no. 10, pp. 2184-2197, October 2010.

[20] G. Zhang, S. Chang, and Z. Alon, “A High Performance Balanced Power Amplifier and Its Integration into a Front-End Module at PCS Band,” IEEE Radio Frequency Integrated Circuits Symposium, June 2007, pp. 251-254. 